Jolanta Socata (Racibórz)

WOJCIECH M. ZAJĄCZKOWSKI (Warszawa)

\title{
LONG TIME EXISTENCE OF SOLUTIONS TO 2D NAVIER-STOKES EQUATIONS WITH HEAT CONVECTION
}

Abstract. Global existence of regular solutions to the Navier-Stokes equations for $(v, p)$ coupled with the heat convection equation for $\theta$ is proved in the two-dimensional case in a bounded domain. We assume the slip boundary conditions for velocity and the Neumann condition for temperature. First an appropriate estimate is shown and next the existence is proved by the Leray-Schauder fixed point theorem. We prove the existence of solutions such that $v, \theta \in W_{s}^{2,1}\left(\Omega^{T}\right), \nabla p \in L_{s}\left(\Omega^{T}\right), s>2$.

1. Introduction. We consider the problem

$$
\begin{array}{ll}
v_{t}+v \cdot \nabla v-\operatorname{div} \boldsymbol{T}(v, p)=\alpha(\theta) g & \text { in } \Omega^{T}=\Omega \times(0, T), \\
\operatorname{div} v=0 & \text { in } \Omega^{T}, \\
\theta_{t}+v \cdot \nabla \theta-\varkappa \Delta \theta=0 & \text { in } \Omega^{T}, \\
\bar{n} \cdot \boldsymbol{D}(v) \cdot \bar{\tau}=0 & \text { on } S^{T}=S \times(0, T), \\
v \cdot \bar{n}=0 & \text { on } S^{T}, \\
\bar{n} \cdot \nabla \theta=0 & \text { on } S^{T}, \\
\left.v\right|_{t=0}=v_{0} & \text { in } \Omega, \\
\left.\theta\right|_{t=0}=\theta_{0} & \text { in } \Omega,
\end{array}
$$

where $\Omega \subset \mathbb{R}^{2}$ is a bounded domain, $\Omega^{T}$ satisfies the weak horn condition (see [2, Sect. 8]) and is not axially symmetric, $S=\partial \Omega \in C^{2}, x=\left(x_{1}, x_{2}\right)$ $\in \mathbb{R}^{2}$ are the Cartesian coordinates, $v=v(x, t)=\left(v_{1}(x, t), v_{2}(x, t)\right) \in \mathbb{R}^{2}$ the

2010 Mathematics Subject Classification: 35Q30, 35Q35, 76D03.

Key words and phrases: Navier-Stokes equations, heat convection, slip boundary condition, Neumann condition, global existence, Leray-Schauder fixed point theorem. 
velocity of the fluid, $\theta=\theta(x, t) \in \mathbb{R}$ the temperature, $\nu>0$ the constant viscosity coefficient, $\varkappa>0$ the constant heat coefficient, $\bar{n}$ the unit outward vector normal to $S$, and $\bar{\tau}$ the unit tangent vector to $S$. By a dot we denote the scalar product in $\mathbb{R}^{2}$.

By $\boldsymbol{D}(v)$ we denote the dilatation tensor of the form

$$
\boldsymbol{D}(v)=\left\{v_{i, x_{j}}+v_{j, x_{i}}\right\}_{i, j=1,2},
$$

and by $g=g(x, t)$ the external force. Finally, $\boldsymbol{T}(v, p)$ denotes the stress tensor of the form

$$
\boldsymbol{T}(v, p)=\nu \boldsymbol{D}(v)-p \boldsymbol{I},
$$

where $\boldsymbol{I}$ is the unit matrix.

Theorem 1.1. Assume that $\alpha \in C^{1}(\mathbb{R})$, there exist constants $c_{2}<c_{3}$ $<\infty$ such that $c_{2} \leq \theta_{0} \leq c_{3}, v_{t}(0) \in L_{2}(\Omega), \theta_{t}(0) \in L_{2}(\Omega), \operatorname{div} v_{0}=0$, $\theta_{0} \in L_{2}(\Omega), 2<s<\eta<\infty, 2<\eta, 4 / s-2 / \eta<1, v_{0} \in W_{s}^{2-2 / s}(\Omega)$, $g \in L_{s}\left(\Omega^{T}\right)$. Moreover assume that $g_{t} \in L_{2}\left(0, T ; L_{l}(\Omega)\right)$, where $l>1$ is arbitrarily close to 1 , and $g \in L_{2}\left(0, T ; L_{r}(\Omega)\right)$, where $r>2$ is arbitrarily close to 2 . Then there exists a solution $(v, p, \theta)$ of problem (1.1) such $v, \theta \in$ $W_{s}^{2,1}\left(\Omega^{T}\right), \nabla p \in L_{s}\left(\Omega^{T}\right)$, and a constant $C_{*}$ such that

$$
\|v\|_{W_{s}^{2,1}\left(\Omega^{T}\right)}+\|\nabla p\|_{L_{s}\left(\Omega^{T}\right)}+\|\theta\|_{W_{s}^{2,1}\left(\Omega^{T}\right)} \leq C_{*},
$$

and

$$
c_{2} \leq \theta \leq c_{3}
$$

2. Notation. Let us consider the Stokes problem

$$
\begin{array}{ll}
v_{t}-\operatorname{div} \boldsymbol{T}(v, p)=f & \text { in } \Omega^{T}, \\
\operatorname{div} v=0 & \text { in } \Omega^{T}, \\
\bar{n} \cdot \boldsymbol{D}(v) \cdot \bar{\tau}=0 & \text { on } S^{T}, \\
v \cdot \bar{n}=0 & \text { on } S^{T}, \\
\left.v\right|_{t=0}=v_{0} & \text { in } \Omega .
\end{array}
$$

ThEOREM 2.1 (proof similar to one in [1]). Let $f \in L_{q}\left(\Omega^{T}\right), v_{0} \in$ $W_{q}^{2-2 / q}(\Omega), \operatorname{div} v_{0}=0, S \in C^{2}, q \in(1, \infty)$. Then there exists a unique solution to problem (2.1) such that $v \in W_{q}^{2,1}\left(\Omega^{T}\right), \nabla p \in L_{q}\left(\Omega^{T}\right)$ and

$$
\|v\|_{W_{q}^{2,1}\left(\Omega^{T}\right)}+\|\nabla p\|_{L_{q}\left(\Omega^{T}\right)} \leq c\left(\|f\|_{L_{q}\left(\Omega^{T}\right)}+\left\|v_{0}\right\|_{W_{q}^{2-2 / q}(\Omega)}\right) .
$$

Theorem 2.2 (see [2, Sect. 10]). Let $\Omega \subset \mathbb{R}^{n}$ be a bounded domain such that $\Omega^{T}$ satisfies the weak horn condition, and let $u \in W_{s}^{2,1}\left(\Omega^{T}\right) \cap L_{2}\left(\Omega^{T}\right)$. Then the following interpolation inequality holds:

$$
\|\nabla u\|_{L_{q}\left(\Omega^{T}\right)} \leq \varepsilon\|u\|_{W_{s}^{2,1}\left(\Omega^{T}\right)}+c(1 / \varepsilon)\|\nabla u\|_{L_{2}\left(\Omega^{T}\right)},
$$


for $s, q \in(1, \infty)$ satisfying $(n+2) / s-(n+2) / q<1, s \leq q$, where $c(a)$ denotes an increasing positive function of a. Moreover, $\varepsilon$ does not depend on $s, n, q$ but $c$ does.

Lemma 2.3 (Korn inequality, see [6]). Assume that $\Omega$ is not invariant with respect to any rotation. Assume that

$$
\|\boldsymbol{D}(v)\|_{L_{2}(\Omega)}<\infty,\left.\quad v \cdot \bar{n}\right|_{S}=0, \quad \operatorname{div} v=0 .
$$

Then there exists a constant $c_{0}$ such that

$$
\|v\|_{H^{1}(\Omega)} \leq c_{0}\|\boldsymbol{D}(v)\|_{L_{2}(\Omega)},
$$

where $c_{0}$ is independent of $v$.

3. Estimates. We show estimates for the temperature.

Lemma 3.1. Assume $\theta(0) \geq c_{2}$. Then for $\theta$ sufficiently regular we have

$$
\theta(t) \geq c_{2} \quad \text { for } t \geq 0 \text {. }
$$

Proof. Let $\left(\theta-c_{2}\right)_{-}=\min \left\{0, \theta-c_{2}\right\}$. Multiplying $(1.3)_{3}$ by $\left(\theta-c_{2}\right)_{-}$ integrating over $\Omega$ and using the boundary conditions we obtain

$$
\frac{1}{2} \frac{d}{d t} \int_{\Omega}\left(\theta-c_{2}\right)_{-}^{2} d x+\varkappa \int_{\Omega}\left|\nabla\left(\theta-c_{2}\right)_{-}\right|^{2} d x=0 .
$$

Integrating with respect to time we have

$$
\frac{1}{2}\left\|\left(\theta-c_{2}\right)_{-}(t)\right\|_{L_{2}(\Omega)}^{2}+\varkappa\left\|\nabla\left(\theta-c_{2}\right)_{-}\right\|_{L_{2}\left(\Omega^{t}\right)}^{2}=\frac{1}{2}\left\|\left(\theta-c_{2}\right)_{-}(0)\right\|_{L_{2}(\Omega)}^{2} .
$$

Since $\left(\theta-c_{2}\right)_{-}(0)=0$ we conclude the proof.

REMARK 3.1. If $\theta(0) \geq 0$, then $\theta(t) \geq 0$ for $t \geq 0$.

Lemma 3.2. Assume $\theta(0) \leq c_{3}$. Then for $\theta$ sufficiently regular we have

$$
\theta(t) \leq c_{3} \quad \text { for } t \geq 0 \text {. }
$$

Proof. Let $\left(\theta-c_{3}\right)_{+}=\max \left\{0, \theta-c_{3}\right\}$. Multiplying $(1.1)_{3}$ by $\left(\theta-c_{3}\right)_{+}$, integrating over $\Omega$, integrating by parts and using the boundary conditions yields

$$
\frac{1}{2} \frac{d}{d t} \int_{\Omega}\left(\theta-c_{3}\right)_{+}^{2} d x+\varkappa \int_{\Omega}\left|\nabla\left(\theta-c_{3}\right)_{+}\right|^{2} d x=0 .
$$

Integrating with respect to time we obtain

$$
\begin{aligned}
\frac{1}{2}\left\|\left(\theta-c_{3}\right)_{+}(t)\right\|_{L_{2}(\Omega)}^{2}+\varkappa \int_{\Omega^{t}}\left|\nabla\left(\theta-c_{3}\right)_{+}\right|^{2} d x d t^{\prime} & \\
& =\frac{1}{2}\left\|\left(\theta_{0}-c_{3}\right)_{+}(0)\right\|_{L_{2}(\Omega)}^{2} .
\end{aligned}
$$

Since $\left(\theta_{0}-c_{3}\right)_{+}(0)=0$ we conclude the proof. 
Lemma 3.3. Assume that $\alpha \in C^{1}(\mathbb{R})$ and there exist constants $c_{2}<$ $c_{3}<\infty$ such that $c_{2} \leq \theta_{0} \leq c_{3}$. Moreover assume that $g_{t} \in L_{2}\left(0, T ; L_{l}(\Omega)\right)$, $l>1, g \in L_{2}\left(0, T ; L_{r}(\Omega)\right), r>2, v_{t}(0) \in L_{2}(\Omega), \theta_{t}(0) \in L_{2}(\Omega), v_{0} \in L_{2}(\Omega)$, $\theta_{0} \in L_{2}(\Omega)$. Then

$$
\begin{aligned}
\|v(t)\|_{L_{2}(\Omega)}^{2}+\nu \int_{0}^{t}\left\|v\left(t^{\prime}\right)\right\|_{H^{1}(\Omega)}^{2} d t^{\prime} \\
\quad \leq c_{1}\|g\|_{L_{2}\left(0, t ; L_{r}(\Omega)\right)}^{2}+\|v(0)\|_{L_{2}(\Omega)}^{2} \equiv c_{4},
\end{aligned}
$$

and

$$
\begin{gathered}
\left\|v_{t}(t)\right\|_{L_{2}(\Omega)}^{2}+\left\|\theta_{t}(t)\right\|_{L_{2}(\Omega)}^{2}+\int_{0}^{t}\left(\left\|v_{t^{\prime}}\right\|_{H^{1}(\Omega)}^{2}+\left\|\theta_{t^{\prime}}\right\|_{H^{1}(\Omega)}^{2}\right) d t^{\prime} \\
\leq c e^{c t} \exp \left[c\left(\left\|v_{0}\right\|_{L_{2}(\Omega)}^{2}+\left\|\theta_{0}\right\|_{L_{2}(\Omega)}^{2}+c_{1}\|g\|_{L_{2}\left(0, t ; L_{r}(\Omega)\right)}^{2}\right)\right] \\
\cdot\left(\left\|v_{t}(0)\right\|_{L_{2}(\Omega)}^{2}+\left\|\theta_{t}(0)\right\|_{L_{2}(\Omega)}^{2}+\left\|g_{t}\right\|_{L_{2}\left(0, t ; L_{l}(\Omega)\right)}^{2}\right) \equiv c_{5} .
\end{gathered}
$$

Proof. Multiply $(1.1)_{1}$ by $v$ and integrate over $\Omega$. Using the boundary conditions and the Korn inequality we obtain

$$
\frac{1}{2} \frac{d}{d t}\|v\|_{L_{2}(\Omega)}^{2}+\frac{\nu}{2}\|v\|_{H^{1}(\Omega)}^{2} \leq c\|\alpha(\theta)\|_{L_{\infty}(\Omega)} \int_{\Omega}|g v| d x .
$$

Since $\alpha \in C^{1}(\mathbb{R})$ Lemmas 3.1 and 3.2 imply that there exists a positive function $\varphi:\left\{(x, y) \in \mathbb{R}^{2}: x \leq y\right\} \rightarrow \mathbb{R}_{+}$such that

$$
\sup _{t}\|\alpha(\theta)\|_{L_{\infty}(\Omega)} \leq \varphi\left(c_{2}, c_{3}\right) .
$$

By the Hölder and Young inequalities, (3.7) and (3.8) imply

$$
\frac{d}{d t}\|v\|_{L_{2}(\Omega)}^{2}+\nu\|v\|_{H^{1}(\Omega)}^{2} \leq c_{1}\|g\|_{L_{r}(\Omega)}^{2},
$$

where

$$
c_{1}=c \varphi^{2}\left(c_{2}, c_{3}\right)
$$

and $r>1$. Integrating (3.9) with respect to time we have (3.5).

Multiplying (1.1) $)_{3}$ by $\theta$, integrating over $\Omega^{T}$ and using the boundary conditions we obtain

$$
\|\theta\|_{L_{2}(\Omega)}^{2}+2 \varkappa\|\nabla \theta\|_{L_{2}\left(\Omega^{t}\right)}^{2}=\left\|\theta_{0}\right\|_{L^{2}(\Omega)}^{2} .
$$

Differentiating $(1.1)_{1}$ with respect to $t$, multiplying by $v_{t}$, integrating over $\Omega$ and then applying the Hölder inequality, an interpolation inequality and the fact that

$$
\left|\alpha_{\theta}(\theta)\right| \leq \varphi_{1}\left(c_{2}, c_{3}\right) \equiv c_{4}
$$


we obtain

$$
\begin{aligned}
\frac{1}{2} \frac{d}{d t}\left\|v_{t}\right\|_{L_{2}(\Omega)}^{2}+ & \frac{\nu}{2}\left\|v_{t}\right\|_{H^{1}(\Omega)}^{2} \\
\leq & c\left[\left\|\nabla v_{t}\right\|_{L_{2}(\Omega)} \cdot\left\|v_{t}\right\|_{L_{2}(\Omega)}+\left\|v_{t}\right\|_{L_{2}(\Omega)}^{2}\right]\|\nabla v\|_{L_{2}(\Omega)} \\
& +c\left\|g_{t}\right\|_{L_{l}(\Omega)}^{2}+c\|g\|_{L_{r}(\Omega)}^{2}\left\|\theta_{t}\right\|_{L_{s}(\Omega)}^{2},
\end{aligned}
$$

where $1 / r+1 / s=1 / \bar{l}$ and $\bar{l}>1$.

Differentiating $(1.1)_{3}$ with respect to $t$, multiplying by $\theta_{t}$, integrating over $\Omega$ and then applying the Hölder inequality and interpolation inequality we have

$$
\begin{aligned}
& \frac{1}{2} \frac{d}{d t} \int_{\Omega} \theta_{t}^{2} d x+\varkappa\left\|\theta_{t}\right\|_{H^{1}(\Omega)}^{2} \leq \int_{\Omega}\left|v_{t}\right|\left|\theta_{t}\right||\nabla \theta| d x+c\left\|\theta_{t}\right\|_{L_{2}(\Omega)}^{2} \\
& \leq\left\|v_{t}\right\|_{L_{4}(\Omega)}\left\|\theta_{t}\right\|_{L_{4}(\Omega)}\|\nabla \theta\|\left\|_{L_{2}(\Omega)}+c\right\| \theta_{t} \|_{L_{2}(\Omega)}^{2} \\
& \leq c\left(\left\|\nabla v_{t}\right\|_{L_{2}(\Omega)}^{1 / 2}\left\|v_{t}\right\|_{L_{2}(\Omega)}^{1 / 2}\left\|\nabla \theta_{t}\right\|_{L_{2}(\Omega)}^{1 / 2}\left\|\theta_{t}\right\|_{L_{2}(\Omega)}^{1 / 2}\right. \\
&+\left\|\nabla v_{t}\right\|_{L_{2}(\Omega)}^{1 / 2}\left\|v_{t}\right\|_{L_{2}(\Omega)}^{1 / 2}\left\|\theta_{t}\right\|_{L_{2}(\Omega)} \\
&+\left\|v_{t}\right\|_{L_{2}(\Omega)}\left\|\nabla \theta_{t}\right\|_{L_{2}(\Omega)}^{1 / 2}\left\|\theta_{t}\right\|_{L_{2}(\Omega)}^{1 / 2} \\
&+\left\|v_{t}\right\|_{L_{2}(\Omega)}\left\|\theta_{t}\right\|_{L_{2}(\Omega)}\|\nabla \theta\|_{L_{2}(\Omega)}+c\left\|\theta_{t}\right\|_{L_{2}(\Omega)}^{2} .
\end{aligned}
$$

Let

$$
\begin{aligned}
& y(t)=\left\|v_{t}\right\|_{L_{2}(\Omega)}^{2}+\left\|\theta_{t}\right\|_{L_{2}(\Omega)}^{2}, \\
& Y(t)=\left\|v_{t}\right\|_{H^{1}(\Omega)}^{2}+\left\|\theta_{t}\right\|_{H^{1}(\Omega)}^{2}, \\
& Z(t)=\|\nabla v\|_{L_{2}(\Omega)}^{2}+\|\nabla \theta\|_{L_{2}(\Omega)}^{2} .
\end{aligned}
$$

Then (3.11) and (3.12) imply

$$
\begin{aligned}
\frac{d}{d t} y+Y \leq & c Y^{1 / 2} y^{1 / 2} Z^{1 / 2} \\
& +c\left(\left\|\theta_{t}\right\|_{L_{2}(\Omega)}^{2}+\left\|g_{t}\right\|_{L_{l}(\Omega)}^{2}+\|g\|_{L_{r}(\Omega)}^{2}\left\|\theta_{t}\right\|_{L_{s}(\Omega)}^{2}\right) .
\end{aligned}
$$

By the Young inequality we get

$$
\frac{d}{d t} y+Y \leq c y Z+c\left(\left\|\theta_{t}\right\|_{L_{2}(\Omega)}^{2}+\left\|g_{t}\right\|_{L_{l}(\Omega)}^{2}+\|g\|_{L_{r}(\Omega)}^{2}\left\|\theta_{t}\right\|_{L_{s}(\Omega)}^{2}\right) .
$$

Setting $s=2$ in (3.14) yields

$$
\frac{d}{d t} y+Y \leq c y\left(Z+1+\|g\|_{L_{r}(\Omega)}^{2}\right)+c\left\|g_{t}\right\|_{L_{l}(\Omega)}^{2}
$$

Integrating (3.15) with respect to time and employing (3.5) and (3.10) we obtain (3.6). 
Lemma 3.4. Let the assumptions of Lemma 3.3 be satisfied. Moreover, assume $v_{0} \in W_{s}^{2-2 / s}(\Omega)$ and $g \in L_{s}\left(\Omega^{T}\right)$ for some $s, 1<s<\infty$. Then

$$
\begin{aligned}
& \|v\|_{W_{s}^{2,1}\left(\Omega^{T}\right)}+\|\nabla p\|_{L_{s}\left(\Omega^{T}\right)}+\|\theta\|_{W_{s}^{2,1}\left(\Omega^{T}\right)} \\
& \quad \leq c\left(\|g\|_{L_{s}\left(\Omega^{T}\right)}+\left\|v_{0}\right\|_{W_{s}^{2-2 / s}(\Omega)}+\left\|\theta_{0}\right\|_{W_{s}^{2-2 / s}(\Omega)}\right)+c\left(c_{4}, c_{5}\right) .
\end{aligned}
$$

Proof. From (3.5) and (3.6) we have

$$
\left\|v_{t}\right\|_{L_{\infty}\left(0, t ; L_{2}(\Omega)\right)}+\|\nabla v\|_{L_{2}\left(\Omega^{t}\right)}+\|v\|_{L_{\infty}\left(0, t ; L_{2}(\Omega)\right)} \leq c\left(c_{4}, c_{5}\right), \quad t \leq T .
$$

Hence $v \in H^{1}\left(\Omega^{T}\right)$. Since $\Omega \subset \mathbb{R}^{2}$ we also have

$$
\|v\|_{L_{6}\left(\Omega^{T}\right)} \leq c\left(c_{4}, c_{5}\right)
$$

Now we want to increase regularity described by (3.17). For this purpose we consider the problem

$$
\begin{aligned}
& v_{t}-\nu \Delta v+\nabla p=-v \cdot \nabla v+\alpha g \\
& \operatorname{div} v=0 \\
& \left.\bar{n} \cdot \boldsymbol{D}(v) \cdot \bar{\tau}\right|_{S^{T}}=0 \\
& \left.v \cdot \bar{n}\right|_{S^{T}}=0 \\
& \left.v\right|_{t=0}=v_{0}
\end{aligned}
$$

To apply Theorem 2.1 we examine

$$
\|v \cdot \nabla v\|_{L_{s}\left(\Omega^{T}\right)} \leq\|v\|_{L_{s \lambda_{1}}\left(\Omega^{T}\right)}\|\nabla v\|_{L_{s \lambda_{2}}\left(\Omega^{T}\right)} \equiv I_{1},
$$

where $1 / \lambda_{1}+1 / \lambda_{2}=1$. Assuming $s \lambda_{1}=6$ we apply (2.3) with $\frac{4}{s}-\frac{4}{s \lambda_{2}}<1$, i.e. $\frac{4}{s \lambda_{1}}<1$, which is satisfied. Hence in view of (3.18) and (2.3) we have

$$
I_{1} \leq c\left(c_{4}, c_{5}\right)\|\nabla v\|_{L_{s \lambda_{2}}\left(\Omega^{T}\right)} \leq \varepsilon\|v\|_{W_{s}^{2,1}\left(\Omega^{T}\right)}+c\left(1 / \varepsilon, c_{4}, c_{5}\right)\|\nabla v\|_{L_{2}\left(\Omega^{T}\right)} .
$$

Assuming that $g \in L_{s}\left(\Omega^{T}\right)$ we apply Theorem 2.1. Then we have

$$
\begin{aligned}
\|v\|_{W_{s}^{2,1}\left(\Omega^{T}\right)}+\|\nabla p\|_{L_{s}\left(\Omega^{T}\right)} \leq & \varepsilon\|v\|_{W_{s}^{2,1}\left(\Omega^{T}\right)}+c\left(1 / \varepsilon, c_{4}, c_{5}\right)\|\nabla v\|_{L_{2}\left(\Omega^{T}\right)} \\
& +c\left(\|\theta\|_{L_{\infty}\left(\Omega^{T}\right)}\right)\|g\|_{L_{s}\left(\Omega^{T}\right)}+c\left\|v_{0}\right\|_{W_{s}^{2-2 / s}(\Omega)} .
\end{aligned}
$$

Assuming that $\varepsilon$ is sufficiently small in view of (3.1) and (3.2) we obtain $v \in W_{s}^{2,1}\left(\Omega^{T}\right)$ for $1<s<6$. Since

$$
\|u\|_{L_{\infty}\left(\Omega^{T}\right)} \leq\|u\|_{W_{s}^{2,1}\left(\Omega^{T}\right)} \quad \text { for } 2<s,
$$

we have $\|v\|_{L_{\infty}\left(\Omega^{T}\right)} \leq c\left(c_{4}, c_{5}\right)$. Assume $1<s<\infty$. To apply Theorem 2.1 we examine

$$
\|v \cdot \nabla v\|_{L_{s}\left(\Omega^{T}\right)} \leq\|v\|_{L_{\infty}\left(\Omega^{T}\right)}\|\nabla v\|_{L_{s}\left(\Omega^{T}\right)} \equiv I_{2}
$$

Hence in view of $(2.3)$ we have

$$
I_{2} \leq c\left(c_{4}, c_{5}\right)\|\nabla v\|_{L_{s}\left(\Omega^{T}\right)} \leq \varepsilon\|v\|_{W_{s}^{2,1}\left(\Omega^{T}\right)}+c\left(1 / \varepsilon, c_{4}, c_{5}\right)\|\nabla v\|_{L_{2}\left(\Omega^{T}\right)} .
$$


Applying Theorem 2.1, assuming that $\varepsilon$ is sufficiently small and using the estimate for $\theta$ we get

$$
\|v\|_{W_{s}^{2,1}\left(\Omega^{T}\right)}+\|\nabla p\|_{L_{s}\left(\Omega^{T}\right)} \leq c\left(\|g\|_{L_{s}\left(\Omega^{T}\right)}+\left\|v_{0}\right\|_{W_{s}^{2-2 / s}(\Omega)}\right)+c\left(c_{4}, c_{5}\right) .
$$

Similarly we obtain estimates for $\theta$ (see [3, Ch. 4, Sect. 9, Th. 9.1], [5, Theorem 17]).

4. Existence. For $\eta>2$ define

$$
\mathcal{M}\left(\Omega^{T}\right)=\left\{(v, \theta) \in\left[L_{\infty}\left(0, T ; W_{\eta}^{1}(\Omega)\right)\right]^{2}\right\} .
$$

Let us consider the problems

$$
\begin{aligned}
& v_{t}-\operatorname{div} \boldsymbol{T}(v, p)=-\lambda[\tilde{v} \cdot \nabla \tilde{v}+\alpha(\tilde{\theta}) g], \\
& \operatorname{div} v=0, \\
& \left.v \cdot \bar{n}\right|_{S}=0,\left.\quad \bar{n} \cdot \boldsymbol{D}(v) \cdot \bar{\tau}\right|_{S}=0, \\
& \left.v\right|_{t=0}=v_{0}
\end{aligned}
$$

and

$$
\begin{aligned}
& \theta_{t}-\varkappa \Delta \theta=-\lambda \tilde{v} \cdot \nabla \tilde{\theta}, \\
& \left.\bar{n} \cdot \nabla \theta\right|_{S}=0, \\
& \left.\theta\right|_{t=0}=\theta_{0},
\end{aligned}
$$

where $\lambda \in[0,1]$ is a parameter and $\tilde{v}, \tilde{\theta}$ are treated as given functions.

Lemma 4.1. Let $\alpha \in C^{1}(\mathbb{R})$. Let

$$
\begin{aligned}
& (\tilde{v}, \tilde{\theta}) \in \mathcal{M}\left(\Omega^{T}\right), \quad \eta>2, \\
& g \in L_{s}\left(\Omega^{T}\right), \\
& v_{0} \in W_{s}^{2-2 / s}(\Omega), \quad 2<s<\eta, \\
& S \in C^{2}, \quad \frac{4}{s}-\frac{2}{\eta}<1 .
\end{aligned}
$$

Then there exists a unique solution to problem (4.1) such that

$$
v \in W_{s}^{2,1}\left(\Omega^{T}\right) \subset L_{\infty}\left(0, T ; W_{\eta}^{1}(\Omega)\right)
$$

and

$$
\begin{aligned}
\|v\|_{L_{\infty}\left(0, T ; W_{\eta}^{1}(\Omega)\right)} \leq & c\|v\|_{W_{s}^{2,1}\left(\Omega^{T}\right)} \leq c\left(\lambda\|(\tilde{v}, \tilde{\theta})\|_{\mathcal{M}\left(\Omega^{T}\right)}^{2}\right. \\
& \left.+\lambda \psi\left(c\|(\tilde{v}, \tilde{\theta})\|_{\mathcal{M}\left(\Omega^{T}\right)}\right)\|g\|_{L_{s}\left(\Omega^{T}\right)}+\left\|v_{0}\right\|_{W_{s}^{2-2 / s}(\Omega)}\right) .
\end{aligned}
$$

Moreover the imbedding $W_{s}^{2,1}\left(\Omega^{T}\right) \subset L_{\infty}\left(0, T ; W_{\eta}^{1}(\Omega)\right)$ is compact for $4 / s-2 / \eta<1$, so for $\eta>2$ we have $s>2$. 
Proof. We have

$$
\begin{aligned}
\|\tilde{v} \cdot \nabla \tilde{v}\|_{L_{s}\left(\Omega^{T}\right)} & \leq c\|\tilde{v}\|_{L_{\infty}\left(\Omega^{T}\right)}\|\nabla \tilde{v}\|_{L_{\eta}\left(\Omega^{T}\right)} \leq c\|\tilde{v}\|_{L_{\infty}\left(0, T ; W_{\eta}^{1}(\Omega)\right)}^{2} \\
& \leq c\|(\tilde{v}, \tilde{\theta})\|_{\mathcal{M}\left(\Omega^{T}\right)}^{2} .
\end{aligned}
$$

Define $\psi:[0, \infty) \rightarrow[0, \infty)$ by

$$
\psi(a)=\sup \{|\alpha(x)|:-a \leq x \leq a\} \quad \text { for } a \geq 0 .
$$

Then

$$
\begin{aligned}
\|\alpha(\tilde{\theta}) g\|_{L_{s}\left(\Omega^{T}\right)} & \leq \psi\left(c\|\tilde{\theta}\|_{L_{\infty}\left(0, T ; W_{\eta}^{1}(\Omega)\right)}\right)\|g\|_{L_{s}\left(\Omega^{T}\right)} \\
& \leq \psi\left(c\|(\tilde{v}, \tilde{\theta})\|_{\mathcal{M}\left(\Omega^{T}\right)}\right)\|g\|_{L_{s}\left(\Omega^{T}\right)} .
\end{aligned}
$$

By Theorem 2.1 the proof is complete.

Lemma 4.2. Assume that

$$
(\tilde{v}, \tilde{\theta}) \in \mathcal{M}\left(\Omega^{T}\right), \quad \eta>2, \quad \eta>s>1, \quad \theta_{0} \in W_{s}^{2-2 / s}(\Omega) .
$$

Then there exists a unique solution to problem (4.2) such that

$$
\theta \in W_{s}^{2,1}\left(\Omega^{T}\right) \subset L_{\infty}\left(0, T ; W_{\eta}^{1}(\Omega)\right)
$$

and

$$
\|\theta\|_{L_{\infty}\left(0, T ; W_{\eta}^{1}(\Omega)\right)} \leq c\|\theta\|_{W_{s}^{2,1}\left(\Omega^{T}\right)} \leq c\left(\lambda\|(\tilde{v}, \tilde{\theta})\|_{\mathcal{M}\left(\Omega^{T}\right)}^{2}+\left\|\theta_{0}\right\|_{W_{s}^{2-2 / s}(\Omega)}\right) .
$$

Proof. We have

$$
\begin{aligned}
\|\tilde{v} \cdot \nabla \tilde{\theta}\|_{L_{s}\left(\Omega^{T}\right)} & \leq\|\tilde{v}\|_{L_{\infty}\left(\Omega^{T}\right)}\|\nabla \tilde{\theta}\|_{L_{\eta}\left(\Omega^{T}\right)} \\
& \leq c\|\tilde{v}\|_{L_{\infty}\left(0, T ; W_{\eta}^{1}(\Omega)\right)}\|\tilde{\theta}\|_{L_{\infty}\left(0, T ; W_{\eta}^{1}(\Omega)\right)} \leq c\|(\tilde{v}, \tilde{\theta})\|_{\mathcal{M}\left(\Omega^{T}\right)}^{2}
\end{aligned}
$$

Then the proof is similar to that of Theorem 9.1 from [3, Ch. 4, Sect. 9] (see also [5, Th. 17]).

To prove the existence of solutions to problem (1.1) we apply the LeraySchauder fixed point theorem (see [4]). Therefore we introduce the mapping $\phi:[0,1] \times \mathcal{M}\left(\Omega^{T}\right) \rightarrow \mathcal{M}\left(\Omega^{T}\right),(\lambda, \tilde{v}, \tilde{\theta}) \mapsto \phi(\lambda, \tilde{v}, \tilde{\theta})=(v, \theta)$, where $(v, \theta)$ is a solution to problems (4.1)-(4.2). For $\lambda=0$ we have the existence of a unique solution. For $\lambda=1$ every fixed point is a solution to problem (1.1).

LEMMA 4.3. Let the assumptions of Lemmas 4.1 and 4.2 be satisfied. Then the mappings $\phi(\lambda, \cdot): \mathcal{M}\left(\Omega^{T}\right) \rightarrow \mathcal{M}\left(\Omega^{T}\right), \lambda \in[0,1]$, are completely continuous.

Proof. By Lemmas 4.1 and 4.2 the mappings $\phi(\lambda, \cdot), \lambda \in[0,1]$, are compact. From this it follows that bounded sets in $\mathcal{M}\left(\Omega^{T}\right)$ are transformed into bounded sets in $\mathcal{M}\left(\Omega^{T}\right)$. Let $\left(\tilde{v}_{i}, \tilde{\theta}_{i}\right) \in \mathcal{M}\left(\Omega^{T}\right), i=1,2$, be two given 
elements. Then $\left(v_{i}, \theta_{i}\right), i=1,2$, are solutions to the problems

$$
\begin{aligned}
& v_{i t}-\operatorname{div} \boldsymbol{T}\left(v_{i}, p_{i}\right)=-\lambda\left(\tilde{v}_{i} \cdot \nabla \tilde{v}_{i}+\alpha\left(\tilde{\theta}_{i}\right) g\right), \\
& \operatorname{div} v_{i}=0, \\
& \left.\bar{n} \cdot \boldsymbol{D}\left(v_{i}\right) \cdot \bar{\tau}\right|_{S}=0,\left.\quad \bar{n} \cdot v_{i}\right|_{S}=0, \\
& \left.v_{i}\right|_{t=0}=v_{0}, \quad i=1,2,
\end{aligned}
$$

and

$$
\begin{aligned}
& \theta_{i t}-\varkappa \Delta \theta_{i}=-\lambda \tilde{v}_{i} \cdot \nabla \tilde{\theta}_{i}, \\
& \left.\bar{n} \cdot \nabla \theta_{i}\right|_{S}=0, \\
& \left.\theta_{i}\right|_{t=0}=\theta_{0}, \quad i=1,2 .
\end{aligned}
$$

To show continuity we introduce the differences

$$
V=v_{1}-v_{2}, \quad P=p_{1}-p_{2}, \quad \mathcal{T}=\theta_{1}-\theta_{2}
$$

which are solutions to the problems

$$
\begin{aligned}
& V_{t}-\operatorname{div} \boldsymbol{T}(V, P)=-\lambda\left[\tilde{V} \cdot \nabla \tilde{v}_{1}+\tilde{v}_{2} \cdot \nabla \tilde{V}+\left(\alpha\left(\tilde{\theta}_{1}\right)-\alpha\left(\tilde{\theta}_{2}\right)\right) g\right] \\
& \operatorname{div} V=0 \\
& \left.V \cdot \bar{n}\right|_{S}=\left.0 \quad \bar{n} \cdot \boldsymbol{D}(V) \cdot \bar{\tau}\right|_{S}=0 \\
& \left.V\right|_{t=0}=0
\end{aligned}
$$

and

$$
\begin{aligned}
& \mathcal{T}_{t}-\varkappa \Delta \mathcal{T}=-\lambda\left[\tilde{V} \cdot \nabla \tilde{\theta}_{1}+\tilde{v}_{2} \cdot \nabla \tilde{\mathcal{T}}\right] \\
& \left.\bar{n} \cdot \nabla \mathcal{T}\right|_{S}=0, \\
& \left.\mathcal{T}\right|_{t=0}=0,
\end{aligned}
$$

where $\tilde{V}=\tilde{v}_{1}-\tilde{v}_{2}, \tilde{\mathcal{T}}=\tilde{\theta}_{1}-\tilde{\theta}_{2}$. Define $\psi_{1}:[0, \infty) \rightarrow[0, \infty)$ by $\psi_{1}(a)=$ $\sup \left\{\left|\alpha^{\prime}(x)\right|:|x| \leq a\right\}$ for $a \geq 0$. In view of [2] and [1,3] we have

$$
\begin{array}{r}
+\left\|\tilde{v}_{2}\right\|_{L_{\infty}\left(\Omega^{T}\right)}\|\nabla \tilde{V}\|_{L_{s}\left(\Omega^{T}\right)}+c \psi_{1}\left(\max \left\{\left\|\tilde{\theta}_{1}\right\|,\left\|\tilde{\theta}_{2}\right\|\right\}\right)\|\tilde{\mathcal{T}}\|_{L_{\infty}\left(\Omega^{T}\right)}\|g\|_{L_{s}\left(\Omega^{T}\right)} \\
\left.+\left\|\tilde{v}_{2}\right\|_{L_{\infty}\left(\Omega^{T}\right)}\|\nabla \tilde{\mathcal{T}}\|_{L_{s}\left(\Omega^{T}\right)}+\|\tilde{V}\|_{L_{\infty}\left(\Omega^{T}\right)}\left\|\nabla \theta_{1}\right\|_{L_{s}\left(\Omega^{T}\right)}\right] \\
\quad \leq c\left(\|\tilde{V}\|_{\mathcal{M}\left(\Omega^{T}\right)}+\|\tilde{\mathcal{T}}\|_{\mathcal{M}\left(\Omega^{T}\right)}\right)
\end{array}
$$

so continuity of $\phi$ follows.

Lemma 4.4. Let the assumptions of Lemmas 4.1 and 4.2 be satisfied. Then for every bounded subset $\mathcal{M}_{0}$ of $\mathcal{M}\left(\Omega^{T}\right)$, the family of maps

$$
\phi(\cdot, \tilde{v}, \tilde{\theta}):[0,1] \rightarrow \mathcal{M}\left(\Omega^{T}\right), \quad(\tilde{v}, \tilde{\theta}) \in \mathcal{M}_{0},
$$

is uniformly equicontinuous. 
Proof. Let $(\tilde{v}, \tilde{\theta}) \in \mathcal{M}_{0}, \lambda_{i} \in[0,1], i=1,2, \lambda_{1} \geq \lambda_{2}$, and $v_{i}, \theta_{i}$ be solutions to the problems

$$
\begin{aligned}
& v_{i t}-\operatorname{div} \boldsymbol{T}\left(v_{i}, p_{i}\right)=-\lambda_{i}(\tilde{v} \cdot \nabla \tilde{v}+\alpha(\tilde{\theta}) g), \\
& \operatorname{div} v_{i}=0, \\
& \left.\bar{n} \cdot \boldsymbol{D}\left(v_{i}\right) \cdot \bar{\tau}\right|_{S}=0,\left.\quad \bar{n} \cdot v_{i}\right|_{S}=0 \\
& \left.v_{i}\right|_{t=0}=v_{0}, \quad i=1,2
\end{aligned}
$$

and

$$
\begin{aligned}
& \theta_{i t}-\varkappa \Delta \theta_{i}=-\lambda_{i} \tilde{v} \cdot \nabla \tilde{\theta}, \\
& \left.\bar{n} \cdot \nabla \theta_{i}\right|_{S}=0, \\
& \left.\theta_{i}\right|_{t=0}=\theta_{0}, \quad i=1,2 .
\end{aligned}
$$

To show uniform equicontinuity we introduce the differences

$$
V=v_{1}-v_{2}, \quad P=p_{1}-p_{2}, \quad \mathcal{T}=\theta_{1}-\theta_{2}
$$

which are solutions to the problems

$$
\begin{aligned}
& V_{t}-\operatorname{div} \boldsymbol{T}(V, P)=-\left(\lambda_{1}-\lambda_{2}\right)(\tilde{v} \cdot \nabla \tilde{v}+\alpha(\tilde{\theta}) g), \\
& \operatorname{div} V=0, \\
& \left.\bar{n} \cdot \boldsymbol{D}(V) \cdot \bar{\tau}\right|_{S}=0,\left.\quad \bar{n} \cdot V\right|_{S}=0, \\
& \left.V\right|_{t=0}=0
\end{aligned}
$$

and

$$
\begin{aligned}
& \mathcal{T}-\varkappa \Delta \mathcal{T}=-\left(\lambda_{1}-\lambda_{2}\right) \tilde{v} \cdot \nabla \tilde{\theta}, \\
& \left.\bar{n} \cdot \nabla \mathcal{T}\right|_{S}=0, \\
& \left.\mathcal{T}\right|_{t=0}=0 .
\end{aligned}
$$

In view of Lemmas 4.1 and 4.2 ,

$$
\begin{aligned}
\|(V, \mathcal{T})\|_{\mathcal{M}\left(\Omega^{T}\right)} \leq & c\left(\left(\lambda_{1}-\lambda_{2}\right)\|(\tilde{v}, \tilde{\theta})\|_{\mathcal{M}\left(\Omega^{T}\right)}^{2}\right. \\
& \left.+\left(\lambda_{1}-\lambda_{2}\right) \psi\left(c\|(\tilde{v}, \tilde{\theta})\|_{\mathcal{M}\left(\Omega^{T}\right)}\right)\|g\|_{L_{s}\left(\Omega^{T}\right)}\right),
\end{aligned}
$$

so uniform equicontinuity of $\phi(\cdot, \tilde{v}, \tilde{\theta})$ follows.

Proof of Theorem 1.1. In view of the above considerations the assumptions of the Leray-Schauder fixed point theorem are satisfied. Hence the main theorem is proved.

\section{References}

[1] W. Alame, On existence of solutions for the nonstationary Stokes system with slip boundary conditions, Appl. Math. (Warsaw) 32 (2005), 195-223.

[2] O. V. Besov, V. P. Il'in and S. M. Nikol'skiı̌, Integral Representations of Functions and Imbedding Theorems, Nauka, Moscow, 1975 (in Russian). 
[3] O. A. Ladyzhenskaya, V. A. Solonnikov and N. N. Ural'tseva, Linear and Quasilinear Equations of Parabolic Type, Nauka, Moscow, 1967 (in Russian).

[4] I. Pawłow and W. M. Zajączkowski, Global existence to a three-dimensional nonlinear thermoelasticity system arising in shape memory materials, Math. Methods. Appl. Sci. 28 (2005), 407-442.

[5] V. A. Solonnikov, A priori estimates for second order parabolic equations, Trudy Mat. Inst. Steklov. 70 (1964), 133-212 (in Russian).

[6] W. M. Zajączkowski, Global existence of axially symmetric solutions to NavierStokes equations with large angular component of velocity, Colloq. Math. 100 (2004), 243-263.

State Higher Vocational School in Racibórz Słowacki St. 55

47-400 Racibórz, Poland

E-mail: jolanta_socala@interia.pl
Institute of Mathematics Polish Academy of Sciences Śniadeckich 8, 00-956 Warszawa, Poland and Institute of Mathematics and Cryptology Cybernetics Faculty Military University of Technology Kaliskiego 2, 00-908 Warszawa, Poland E-mail:.wz@impan.pl

Received on 2.7.2008;

revised version on 3.3.2009 
